\title{
Effect of Varied Gradation Levels of Noise Reduction Algorithm on Speech Perception through Acoustical and Perceptual Measures
}

\author{
Sharath Kumar KS* and Manjula P \\ Department of Audiology, All India Institute of Speech and Hearing (AIISH), University of Mysuru, India
}

Submission: March 05, 2016; Published: March 16, 2017

*Corresponding author: Sharath Kumar KS, Department of Audiology (New JC block), Manasagangotri, AIISH, Mysuru - 570 006, Karnataka, India, Tel: +919845421986; Email: sharathkumarks08@gmail.com

Second author: Manjula P, Professor of Audiology, Department of Audiology, Manasagangotri, AIISH, Mysuru - 570006 India, Karnataka, Tel: +919731251948; Email: manjulap21@hotmail.com

\begin{abstract}
Purpose: The purpose of the study was to evaluate the effect of different gradations of noise reduction (NR) algorithm in hearing aids, through acoustical and perceptual measures.

Methods: The output of the hearing aid with NR at three gradations (minimum, medium and maximum) and without NR, at $0 \mathrm{~dB}$ signal to noise ratio (SNR) across three types of noise (cafeteria, speech babble, and traffic noise) was recorded. The effect of noise reduction was quantified through objective and subjective measures.

Results: In noise alone condition, the maximum gain reduction was seen for the traffic noise followed by cafeteria noise and speech babble. In the speech in noise condition, the maximum improvement in the output SNR was seen only for cafeteria noise and traffic noise. However, the NR gradation did not have much impact on speech babble. The Perceptual Evaluation of Speech Quality results revealed that the mean opinion scores were similar across the NR 'on' and NR 'off' positions. However, Envelope Detection Index values for maximum NR gradation was better than medium and minimum gradation for cafeteria noise and traffic noise. Hence, it was seen that the effectiveness of NR depended on the type of noise. The subjective quality judgment tasks were also in coherence with the objective measures.
\end{abstract}

Conclusion: When NR was set to maximum, it could aid in reducing the noisiness and improving the overall quality of the signal for cafeteria and traffic noise.

Keywords: Acoustic analysis; EDI; Hearing aid; Noise reduction algorithm - gradation; PESQ; Quality judgment; WADA-SNR

Abbreviations: NR: Noise Reduction; SNR: Signal-to-Noise Ratio; WADA-SNR: Waveform Amplitude Distribution Analysis-Signal to Noise Ratio; EDI: Envelope Difference Index; PESQ: Perceptual Evaluation of Speech Quality

\section{Introduction}

Digitization has revolutionized the hearing aid technology in the recent times. One such revolution includes the noise reduction (NR) algorithm. The NR feature was available in the hearing aids since the 1970s in the form of tone control switch (H or N-L) to cut-off the low frequencies [1]. Since then many attempts have been made to improvise the algorithms to reduce the background noise in the hearing aids. Customer satisfaction from MarkeTrak VII indicates that only $51 \%$ of individuals are satisfied using the hearing aid in the presence of noise [2].

The noise reduction algorithms utilize decision rules that define what constitutes noise, how much reduction is required in gain, and in which frequency region the reduction in gain should be applied. The noise reduction algorithms in the present day hearing aids usually are modulation based. These algorithms analyze and keep the incoming speech (that which changes in level over time) constant and reduce noise (that which is steady over time). Very few of the NR algorithms use subtraction techniques [1]. The frequency importance function of the NR algorithm that is used to shape the maximum gain reduction may not be same across channels. That is, some hearing aids may have equal reduction across all the channels while others may not have the gain reduction at frequencies that are important for speech [3]. 
The NR feature has been added with different gradations in the recent hearing aids. The gradation indicates the amount of noise reduction feature (in percent) that is being acted upon to process the input signal. This can be varied by adjusting the 'strength'/ 'gradation' available in the hearing aid during programming. The maximum gain reduction can be around 20 $\mathrm{dB}$ at the maximum gradation / stronger setting in some hearing aids $[1,3,4]$, and can be only $5 \mathrm{~dB}$ for the minimum gradation / milder setting $[1,3,5,6]$.

Rickets and Hornsby [4] evaluated the effect of digital noise reduction (DNR) processing on aided speech recognition and sound quality measures. They reported that participants preferred the DNR 'on' condition (gradation set to maximum) significantly more often than the DNR 'off' condition. Zakis et al. [7] evaluated the various configurations of environmental noise reduction (ENR) algorithms. The gain reduction was simulated to provide a $10 \mathrm{~dB}$ reduction in all channels (ENR StrongFlat), similar to a stronger noise-reduction setting and 2-10 dB across channels (ENR MildSII) with a modulation depth of $0 \mathrm{~dB}$, which was similar to that available in some of the commercial hearing aids. ENR StrongFlat setting focused on listening comfort whereas, ENR MildSII focused on speech understanding. The results showed that the average reduction of output-level across channels for speech-shaped noise was 3.9 and $9.4 \mathrm{~dB}$; that for eight-talker babble was 1.8 and $3.1 \mathrm{~dB}$ for ENR MildSII and ENR StrongFlat, respectively. They also noted that for the eight-talker babble, the differences between ENR MildSII and ENR StrongFlat were reduced. Also, with both configurations provided less suppression of babble noise than speech-shaped white noise.

In an attempt to reduce noise in the hearing aid processing, the NR should not compromise on the quality of the perceived speech. The way different levels of gradation would aid in reducing the noise of the input the signal has been studied sparsely. An investigation into this is important because the quality of the speech signal should not be altered at maximum gradation levels and cause distortion at the output. It may also happen that the NR at minimum gradation may not bring sufficient noise reduction, as required for an individual. Hence, it becomes important to evaluate the effect of different NR gradations. This would help in selecting the appropriate gradation level for an individual. Thus, the present study attempts to study the effect of different gradation levels of NR in hearing aids, through acoustical and perceptual measures.

\section{Materials and Methods}

The aim of the study was to evaluate the effect of different gradation levels of noise reduction (NR) algorithm in hearing aids, through acoustical and perceptual measures. The following methods were adopted to study this.

\section{Procedure}

Kannada sentence lists [8] were used as speech stimuli. Three types of noise were included, viz., speech babble (eight- talker speech babble recorded by Anitha and Manjula, [9]), traffic noise and cafeteria noise. The experiment was conducted in two phases. Phase 1 involved studying the effect of gradation levels in NR through acoustical measures; Phase 2 involved studying the effect of gradation levels in NR through perceptual measures.

Phase 1: In Phase 1, the effect of gradation levels in NR was studied through acoustical measures. A commercially available digital Receiver-in-canal (RIC) hearing aid with twelve channels and a fitting range from mild to moderately-severe degree of hearing loss was selected. The selected hearing aid had NR feature which utilized a modulation based noise detection and channel-wise noise reduction facility. The hearing aid was programmed through NOAHLink, using the hearing aid specific software. The audiogram was simulated to mimic a hearing loss with $50 \mathrm{~dB}$ HL as thresholds for both air-conduction and bone-conduction, at all audiometric frequencies. The hearing aid was programmed using the NAL-NL2 prescriptive formula. The hearing aid programming was set to the new hearing aid user with acclimatization level set to non-experienced user at the time of fitting. The 'first fit' was applied for setting the hearing aid parameters. The compression in the hearing aid was disabled. The microphone was set to omni-directional mode. This programmed RIC was considered for recording the output of the hearing aid when fitted on the ear of the Knowles Electronic Manikin for Acoustic Research (KEMAR).

Instrumental set-up for recording the output from the hearing aid: The G.R.A.S. 45BB KEMAR with the ear simulator RA0045 was placed at the center of the test room. The programmed RIC hearing aid was placed on the pinna of the KEMAR and the hearing aid was connected to the canal with a double dome tip. The signal was generated by a personal computer and the stimulus was presented through a loudspeaker that was mounted in front at a distance of one meter and $0^{\circ}$ azimuth from the KEMAR. The output of the hearing aid was picked up using an ear simulator RA0045 microphone (Type $40 \mathrm{AG}$ ) located in the KEMAR and was monitored, recorded and stored using the sound analyzer (Brüel \& Kjær Type 2270). The level of the input signal was also monitored through Brüel\&Kjær Type 2270 sound analyzer during the recording process.

The output from the hearing aid was recorded in the three conditions. They were:

a. Speech Alone Condition: Single Kannada sentence was presented at $65 \mathrm{~dB}$ SPL to KEMAR and the output from the ear (single sentence as heard from the canal of the KEMAR was recorded) of the KEMAR was recorded without the hearing aid. This served as the reference 'unprocessed speech' for comparison with the two aided conditions.

b. Noise Alone Condition: Cafeteria noise, speech babble, and traffic noise were given individually as input to the hearing aid fitted to the KEMAR, at $65 \mathrm{~dB}$ SPL. The hearing aid output at the ear of the KEMAR was recorded with NR of the 
hearing aid in 'on' and 'off' conditions. In NR 'on' condition, the NR gradation was kept at minimum (NR min), medium (NR med) and maximum (NR max) position and outputs were recorded separately.

c. Speech in Noise Condition: A single sentence and noise were presented simultaneously through the loudspeaker kept at $0^{\circ}$ Azimuth at one-meter distance from the KEMAR. The noise was presented for 10 seconds before the onset of speech in order to activate the noise reduction algorithm in the hearing aid. This was repeated for each of the different types of noise at $0 \mathrm{~dB}$ SNR. The output of the hearing aid, with NR 'on' and 'off', in the ear of the KEMAR was recorded for analysis. In NR 'on' condition, the NR gradation was kept at minimum, medium and maximum position, and the outputs were recorded separately.

Phase 2: Phase 2 involved studying the effect of gradation levels in NR through perceptual measures. Twenty-one participants between 20 and 24 years of age (21.76 years) participated in the study. All participants had normal hearing sensitivity with pure tone thresholds less than or equal $15 \mathrm{~dB}$ $\mathrm{HL}$ at $0.25 \mathrm{kHz}, 0.5 \mathrm{kHz}, 1 \mathrm{kHz}, 2 \mathrm{kHz}, 4 \mathrm{kHz}$ and $8 \mathrm{kHz}$ for airconduction and less than or equal to $15 \mathrm{~dB} \mathrm{HL}$ at $0.25 \mathrm{kHz}, 0.5$ $\mathrm{kHz}, 1 \mathrm{kHz}, 2 \mathrm{kHz}$ and $4 \mathrm{kHz}$ for bone-conduction in both ears. The participants considered for the study had speech identification scores (SIS) at $40 \mathrm{~dB}$ SL (re: SRT) of $>85 \%$ for both ears. The ears had a normal middle ear function and presence of acoustic reflex at $0.5 \mathrm{kHz}, 1 \mathrm{kHz}, 2 \mathrm{kHz}$ and $4 \mathrm{kHz}$. All participants had normal speech and language skills and were native speakers of Kannada language. The participants had a minimum of higher secondary education, i.e., matriculation or equivalent. Participants having any complaint or history of psychological and neurological problems were excluded.

a. Noise alone condition: The recorded noise from the hearing aid was presented individually through a personal computer using Adobe Audition software. The participant was made to sit comfortably and the recorded output from the hearing aid was presented through headphones monaurally. The data presented here was a part of the doctoral thesis. Since the testing was also done on individuals with hearing impairment with monaural hearing aid fitting for the thesis, testing was done monaurally on individuals with normal hearing in the present study. Pair-wise comparisons were made between the three noise types and three NR gradations. This gave rise to six pairs for comparison within each type of noise (e.g., NR-off with NR-maximum, NR-off with NR-medium, NR-medium with NRminimum etc.). The participant was instructed to judge which sample in the pair was less noisy. Five runs were taken for each pair and average of only the last three runs out of five responses were considered for the judgment. This was considered as Loudness Judgment for Noise (LJN).

b. Speech in noise condition. The recorded speech in noise (single sentence with noise) from the hearing aid was presented individually through a personal computer using Adobe Audition software. The participant was made to sit comfortably and the recorded output from the hearing aid was presented through headphones monaurally (a justified in noise alone condition). Pair-wise comparisons were made between the three noise types and three NR gradations as described in noise alone condition. Participants were made to compare the pairs and the overall clarity of speech (denoted as QJC- Quality Judgement for Clarity); noisiness (denoted as QJN- Quality Judgement for Noisiness) and overall preference (denoted as OP).

For measuring the clarity of speech (QJC), the participants were asked to concentrate on speech signal in the presence of noise, and judge which sample in the pair had better speech clarity in terms of intelligibility. For measuring noisiness (QJN), the participants were asked to concentrate on noise and judge which sample in the pair was less noisy. Lastly, the participants were asked to choose a sample in the pair which they prefer to hear in terms of the overall quality (OP) of the signal. Five runs were taken for each pair and average of only the last three runs out of five responses were considered in the judgment for each of the measures.

\section{Analysis}

The acoustic analysis of the hearing aid output was measured in three conditions. They are given below:

a. Noise alone condition: The recorded hearing aid output of the cafeteria noise, speech babble, traffic noise were analyzed using the virtual Sound Level Meter (SLM), a MATLAB code developed by Lanman [10]. Total LEQ in $\mathrm{dB}$ (A) and LEQ at the 90th percentile were calculated for each type of noise. This is the sound pressure level of noise that exceeded $90 \%$ of the measurement time. i.e., for $90 \%$ of the time, the noise level is above this measured level. The analysis of hearing aid output in the noise alone condition, with NR'on' and 'off' setting, was done for the final 40 seconds duration out of the total 50 seconds of stimulus duration.

b. Speech in noise condition: WADA-SNR, PESQ, and EDI were computed using the following procedure. Waveform Amplitude Distribution Analysis (WADA), given by Kim and Stern [11], was used to estimate the SNR, henceforth referred to as WADA-SNR. The measurement procedure was incorporated into a MATLAB code (version 0.3) developed by Ellis [12]. This code automatically calculates the SNR in $\mathrm{dB}$ by comparing the sentences recorded in the presence of noise, while the NR was 'on' (at different gradations) and NR 'off'. Perceptual Evaluation of Speech Quality (PESQ) is an objective method for predicting the subjective quality of speech. This was developed by the International Telephone Union (ITU) for assessing the sound quality in telephone systems. The PESQ predicts how close the target signal is in terms of its quality, in comparison to the original signal. The PESQ provides a value between 0 and 4.5. If 
the value is 4.5 , then the target speech is same as unprocessed speech in terms of quality. This measurement procedure was incorporated into a MATLAB code (version 0.3) developed by Ellis [12]. This code automatically provided the mean opinion scores (MOS) for PESQ. Envelope Difference Index (EDI) is a non-standardized MATLAB code based on the method given Statistical analysis

Table 1: Illustration of data arrangement for performing the McNemar test.

\begin{tabular}{|c|c|c|c|c|}
\hline \multirow{2}{*}{ E.g. Pair } & \multirow{2}{*}{$\begin{array}{l}\text { Choice given by } \\
\text { researcher }\end{array}$} & \multirow{2}{*}{ Participant's choice } & \multicolumn{2}{|c|}{ Pattern of scoring } \\
\hline & & & NR off & NR max \\
\hline NR off - NR max & $\begin{array}{c}\text { NR off/ NR max/ both } \\
\text { are same }\end{array}$ & NR off & 1 & 0 \\
\hline NR off - NR max & $\begin{array}{c}\text { NR off/ NR max/ both } \\
\text { are same }\end{array}$ & NR max & 0 & 1 \\
\hline NR off - NR max & $\begin{array}{c}\text { NR off/ NR max/ both } \\
\text { are same }\end{array}$ & Same & 1 & 1 \\
\hline
\end{tabular}

Since a single sentence was used for the acoustic analysis, the statistical test could not be administered for phase 1 , in order to test the difference between the different conditions (across the NR gradations and types of noise). However, for phase 2, non-parametric McNemar test was performed individually on each pair across the three types of noise and quality judgment tasks. SPSS (ver.20) was used to perform that statistical analysis. Since the data obtained from phase 2 were nominal, a nonparametric test was indicated. In addition, the response pattern obtained from the participants was to choose one among the three options provided by the researcher. Hence, to analyze this data, McNemar test was chosen. The raw data were further rearranged to perform the McNemar test. The following Table 1 illustrates the same.

\section{Results}

Results have been enumerated for noise alone and speech in noise conditions under phase 1 (acoustical analysis) and phase 2 (perceptual analysis).

\section{Phase 1 (Acoustical measures)}

In phase 1, for noise alone condition, LEQ was measured and for speech in noise condition, WADA-SNR, PESQ, and EDI analysis were done to understand the output SNR, perceptual quality and temporal variations of the input signal.

a. Noise alone condition: The hearing aid output for noise alone condition for three types of noise, i.e., cafeteria, speech babble, and traffic noise, were analyzed for overall LEQ and LEQ at the 90th percentile in dB (A), with NR algorithm 'off' and NR algorithm 'on' across three gradations. Figure 1 illustrates the LEQ at the 90th percentile in $\mathrm{dB}(\mathrm{A})$. by Fortune et al. [13]. The EDI, being a temporal measure, was used to evaluate how close the two signals are in terms of their envelopes. The EDI values range from 0 to 1 . The value of 0 indicates perfectly similar envelopes and the value of 1 indicates completely dissimilar envelopes.
From Figure 1, it can be noted that the 90th percentile LEQ values in $\mathrm{dB}(\mathrm{A})$ for NR algorithm 'on' condition was lower than that for NR algorithm 'off' condition, for all types of noise. The NR gradation at maximum (NR max) had a greater gain reduction of noise than medium (NR med) followed by minimum (NR min) gradations. The NR min had the least effect on the noise, i.e., NR 'on' at minimum gradation was almost equivalent to NR 'off' position across all the types of noise. The maximum gain reduction (the difference between NR 'off' and NR max) was seen for the traffic noise $(6.9 \mathrm{~dB})$ followed by cafeteria noise $(4.6$ $\mathrm{dB}$ ) and speech babble ( $1.5 \mathrm{~dB})$. From this, it can be deduced that there is an effective reduction in the level of noise when NR of the hearing aid was enabled. This reduction is more effective at NR max than at medium or minimum gradations.

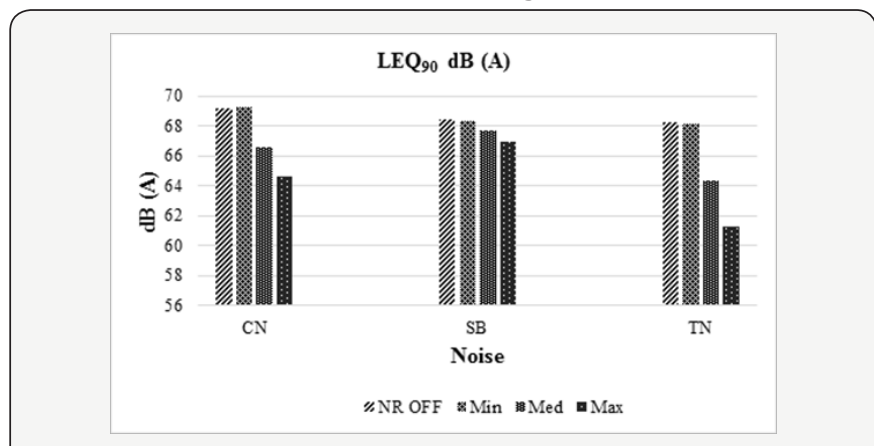

Figure 1: $90^{\text {th }}$ percentile LEQ (dB A) with NR 'off' and NR 'on' across three gradations for cafeteria noise $(\mathrm{CN})$, speech babble $(\mathrm{SB})$ and traffic noise $(\mathrm{TN})$.

b. Speech in noise condition: The acoustic analysis of a sentence with NR 'off' and 'on' across minimum, medium and maximum gradations and three types of noise at $0 \mathrm{~dB}$ input SNR done. The results of the hearing aid output analyzed using 
WADA-SNR tool to measure the output SNR, PESQ to assess the subjective quality of speech and EDI to assess the temporal variations in the output are explained below. The following are the results of speech in noise condition assessed through WADASNR between NR 'off' and 'on' across minimum, medium and maximum gradations for three different types of noise at $0 \mathrm{~dB}$ SNR. Figure 2 illustrates the WADA-SNR output across three NR gradations of cafeteria noise, speech babble, and traffic noise.

From Figure 2 it can be seen that the output SNR was better (increased) with NR 'on' as against NR 'off' condition of all the types of noise. Among the three NR gradations, maximum NR gradation showed better output SNR than medium and minimum gradations (i.e, the SNR maximum $>$ medium $>$ minimum). The minimum NR gradation had the least effect on the noise, i.e., NR 'on' at minimum gradation was almost equivalent to NR 'off' position across all the types of noise. Maximum improvement (the difference between NR 'off' and maximum NR gradation) in the output SNR was seen only for cafeteria noise $(5.15 \mathrm{~dB})$ and traffic noise (5.83 dB). However, the NR gradation did not have much impact on speech babble (0.72 dB).

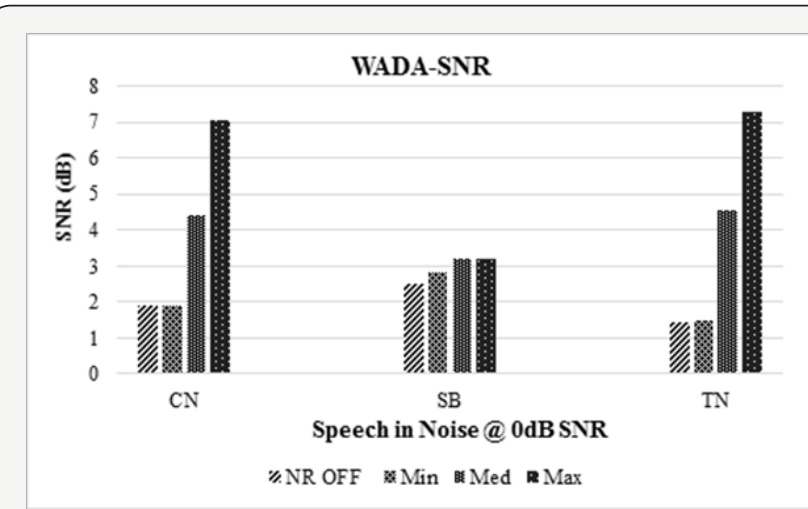

Figure 2: WADA-SNR for NR 'off' and NR 'on' across three gradations for cafeteria noise $(\mathrm{CN})$, speech babble $(\mathrm{SB})$ and traffic noise (TN).

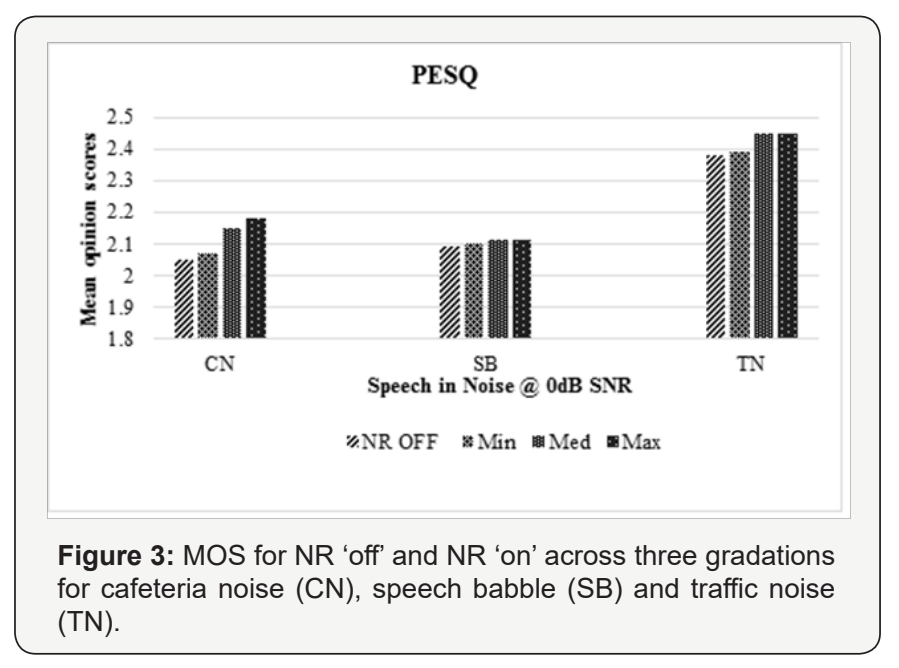

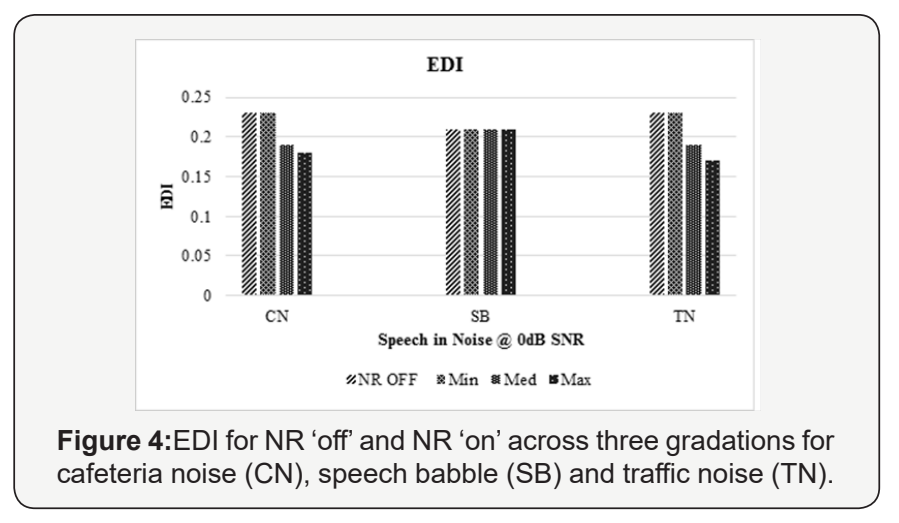

The PESQ results revealed that the mean opinion scores (MOS) were similar across the NR 'on' and NR 'off' positions. However, for $\mathrm{CN}$ and TN, the NR 'on' at maximum gradation was slightly better than NR 'off' condition. Since a single sentence was used for the analysis, the statistical test could not be administered in order to test if the difference in MOS of NR max and NR 'off' conditions of CN and TN were significant. On the whole, the traffic noise had slightly better MOS than the other two types of noise. The MOS for an input SNR of $0 \mathrm{~dB}$ SNR across the three NR gradation and three types of noise is shown in Figure 3. The results from EDI revealed that temporal variations of the speech in noise signal were better preserved in the NR 'on' position as against NR 'off' position. Figure 4 illustrates the results of EDI.

The maximum NR gradation had better EDI values than medium and minimum gradation for cafeteria noise $(0.18)$ and traffic noise (0.17). This illustrates that the temporal variations of the speech in noise signal were better preserved at maximum NR gradation. The minimum NR gradation had least effect on the noise, i.e., NR 'on' at minimum gradation was almost equivalent to NR 'off' position across all the types of noise. However, the EDI values were almost similar across all NR gradations for speech babble, i.e., the NR did not have an impact on speech babble.

\section{Phase 2 (Perceptual measures)}

The results of perceptual quality rating have been enumerated in this section. As it can be recalled, pair-wise comparisons were made between the three noise types and three NR gradations. Non-parametric McNemar test was performed individually on each pair across the three types of noise and quality judgment tasks. The results of McNemar test are given in Table1. The table also illustrates the percentage (\%) of participants (in parentheses) preferring one among the pair (when statistically significant) in the judgment task.

E.g.: For the NR off- NR min pair, only $\mathrm{CN}$, and TN had statistically significant difference in the LJN task. This implies that there was the difference in loudness of noise between the NR off and NR min condition for CN and TN. When the raw data were analyzed, $42 \%$ of the participants had preferred NR min for $\mathrm{CN}$ and $47 \%$ of the participants had preferred NR min for TN in the LJN task. On similar lines, if the pair is significant in a QJC 
task, then it implies that there was a difference in the quality of speech in the presence of noise between the pairs. If a pair is significant in QJN task, then there is a difference in the quality of noisiness between the pairs and in the OP there is a difference in both noisiness and overall clarity of the stimulus.

When the data were compared across the gradation, NR max was chosen whenever it was compared with NR off, NR min and
NR med; NR med was chosen whenever it was compared with NR min. Further, there was no significant difference between the pairs for speech babble across all judgment tasks. However, loudness judgment in noise only condition (between the pairs NR off - NR max and NR max - NR min) for speech babble were not statistically significant. Similar results were also seen for judgment of noisiness in the speech in noise condition (only between the pair NR off - NR max) for speech babble (Table 2).

Table 2: Results of the McNemar test for the quality judgment task for each NR pair across the three types of noise and percentage (\%) of participants (in parenthesis) preferring one among the pair (when significant) in the judgment task.

\begin{tabular}{|c|c|c|c|c|c|c|c|c|c|c|c|c|}
\hline \multirow{2}{*}{$\begin{array}{l}\text { Aided } \\
\text { conditions }\end{array}$} & \multicolumn{3}{|c|}{ LJN } & \multicolumn{3}{|c|}{ QJC } & \multicolumn{3}{|c|}{ QJN } & \multicolumn{3}{|c|}{ OP } \\
\hline & CN & SB & TN & CN & SB & TN & CN & SB & TN & $\mathrm{CN}$ & SB & TN \\
\hline NR off- NR min & $\begin{array}{c}\mathrm{S} \\
(42 \%) \\
@\end{array}$ & NS & $\begin{array}{c}\text { S } \\
(47 \%) \\
@\end{array}$ & NS & NS & NS & NS & NS & NS & NS & NS & NS \\
\hline NR off- NR med & $\begin{array}{c}S \\
(100 \%) \\
\#\end{array}$ & NS & $\begin{array}{c}S \\
(95 \%) \\
\#\end{array}$ & $\begin{array}{c}S \\
(85 \%) \\
\#\end{array}$ & NS & $\begin{array}{c}\mathrm{S} \\
(90 \%) \\
\#\end{array}$ & $\begin{array}{c}S \\
(85 \%) \\
\#\end{array}$ & NS & $\begin{array}{c}\text { S } \\
(95 \%) \\
\#\end{array}$ & $\begin{array}{c}S \\
(76 \%) \\
\#\end{array}$ & NS & $\begin{array}{c}S \\
(85 \%) \\
\#\end{array}$ \\
\hline NR off- NR max & $\begin{array}{c}\mathrm{S} \\
(100 \%) \\
*\end{array}$ & $\begin{array}{c}S \\
(66 \%)^{*}\end{array}$ & $\begin{array}{c}\mathrm{S} \\
(100 \%) \\
*\end{array}$ & $\begin{array}{c}\mathrm{S} \\
(100 \%) \\
*\end{array}$ & NS & $\begin{array}{c}S \\
(100 \%) \\
*\end{array}$ & $\begin{array}{c}S \\
(95 \%) \\
*\end{array}$ & $\begin{array}{c}\mathrm{S} \\
(57 \%) \\
*\end{array}$ & $\begin{array}{c}\mathrm{S} \\
(100 \%) \\
*\end{array}$ & $\begin{array}{c}\text { S } \\
(100 \%) \\
*\end{array}$ & NS & $\begin{array}{c}\text { S } \\
(100 \%) \\
*\end{array}$ \\
\hline NR max- NR min & $\begin{array}{c}\mathrm{S} \\
(100 \%) \\
*\end{array}$ & $\begin{array}{c}\mathrm{S} \\
(38 \%)^{*}\end{array}$ & $\begin{array}{c}\mathrm{S} \\
(100 \%) \\
*\end{array}$ & $\begin{array}{c}S \\
(100 \%) \\
*\end{array}$ & NS & $\begin{array}{c}S \\
(100 \%) \\
*\end{array}$ & $\begin{array}{c}\mathrm{S} \\
(100 \%) \\
*\end{array}$ & NS & $\begin{array}{c}\mathrm{S} \\
(100 \%) \\
*\end{array}$ & $\begin{array}{c}\mathrm{S} \\
(100 \%) \\
*\end{array}$ & NS & $\begin{array}{c}\mathrm{S} \\
(100 \%) \\
*\end{array}$ \\
\hline NR max- NR med & $\begin{array}{c}\mathrm{S} \\
(100 \%) \\
*\end{array}$ & NS & $\begin{array}{c}\mathrm{S} \\
(100 \%) \\
*\end{array}$ & $\begin{array}{c}\mathrm{S} \\
(95 \%) \\
*\end{array}$ & NS & $\begin{array}{c}\mathrm{S} \\
(100 \%) \\
*\end{array}$ & $\begin{array}{c}\mathrm{S} \\
(95 \%) \\
*\end{array}$ & NS & $\begin{array}{c}\mathrm{S} \\
(100 \%) \\
*\end{array}$ & $\begin{array}{c}\mathrm{S} \\
(95 \%) \\
*\end{array}$ & NS & $\begin{array}{c}\mathrm{S} \\
(100 \%) \\
*\end{array}$ \\
\hline NR med- NR min & $\begin{array}{c}S \\
(76 \%) \\
\#\end{array}$ & NS & $\begin{array}{c}S \\
(85 \%) \\
\#\end{array}$ & $\begin{array}{c}S \\
(47 \%) \\
\#\end{array}$ & NS & $\begin{array}{c}S \\
(76 \%) \\
\#\end{array}$ & $\begin{array}{c}\mathrm{S} \\
(95 \%) \\
*\end{array}$ & NS & S & S & NS & S \\
\hline
\end{tabular}

Note: S-significant difference ( $p>0.05)$, NS- No significant difference $(p<0.05)$;

@ - \% preferring NR min; \#- \% preferring NR med; *- \% preferring NR max.

The difference percentage of preference across all the pairs implies that participants had judged both samples as 'same

\section{Discussion}

The discussion has been deliberated for phase 1 (acoustical measures) and phase 2 (perceptual measures).

\section{Phase 1 (Acoustical measures)}

a. Noise only condition: Results from the noise alone condition revealed that there is an effective reduction in the level of noise when NR of the hearing aid was enabled and this reduction was more effective at maximum NR gradation than at medium or minimum gradations. The maximum gain reduction seen was about $6.9 \mathrm{~dB}$ for TN (between NR off and NR max) in the present study. However, Chung [3], Ricketts and Hornsby [4] and Bentler and Chiou [1] have reported that the maximum gain reduction can be around $20 \mathrm{~dB}$ at the maximum gradation/ stronger setting in some hearing aids. On the other hand, there can be only $5 \mathrm{~dB}$ reduction for the minimum gradation/ milder setting [1,3,5,6]. Ricketts and Hornsby [4] reported that in practice, gain reduction for a $70 \mathrm{~dB}$ SPL input using the maximum setting varies from 0 to $14 \mathrm{~dB}$ and it specifically depends on the modulation rate and modulation depth of the input signal.

b. Speech in noise condition: Results of acoustical analysis of speech in noise revealed that the maximum improvement (the difference between NR 'off' and maximum NR gradation) in the output SNR was seen only for cafeteria noise ( $5.15 \mathrm{~dB})$ and traffic noise (5.83 dB). However, the NR gradation did not have an impact on speech babble $(0.72 \mathrm{~dB})$. This could be due to the fact that the other types of noise had nonspeech like characteristics and speech babble had speech-like characteristics. It is known that clean speech has considerably more amplitude fluctuations than speech in a babble or speech in speech-shaped noise [1], hence making a modulation based 
algorithm to differentiate speech and noise would be difficult. The PESQ scores were also similar across the NR gradations. However, on the whole, the traffic noise had slightly better scores than the other two types of noise. It may be suggested that there is a need for a different type of NR specially designed for speech babble. Also, situation and noise specific NR algorithms may provide better comfort and better performance in noisy situations.

The EDI values revealed that the maximum NR gradation was better than medium and minimum gradation for cafeteria noise $(0.18)$ and traffic noise $(0.17)$. This indicates that the temporal variations of the speech in noise signal were better preserved even at maximum NR gradation. The minimum NR gradation had the least effect on the noise, i.e., NR 'on' at minimum gradation was almost equivalent to NR 'off' position across all the types of noise. Geetha and Manjula [14] suggested that compression, DNR, and directionality are similar in terms of temporal changes that they introduce. Hence the results of EDI with manipulating the compression parameter may be utilized to compare with the EDI results while manipulating the NR algorithm. Walaszek [15] evaluated temporal contrast of the speech envelope before and after the compression using EDI.

Danish sentences in the presence of a single female talker background noise or in the presence of the International Collegium of Rehabilitative Audiology (ICRA) noise were used as stimuli. Results revealed that the mean EDIs varied from 0.12 to 0.22 and this depends on the type of background noise. The authors observed that the values are generally higher for the background of the one-talker speech than in the background of two- talker modulated speech-shaped noise. Similar results were seen in the present study, i.e., EDI values were almost similar across all NR gradations for speech babble, i.e., the NR did not have an impact on speech babble. Chung [3] hypothesized that activating NR in the WDRC hearing aid might result in a better speech transmission index and they attributed this enhancement in the temporal envelope to the NR algorithm. Hence, EDI may be one of the tools to understand the temporal variations of a signal processed through the hearing aid.

\section{Phase 2 (Perceptual measures)}

The pair-wise comparisons were made between the NR 'off' and other NR 'on' conditions (across gradations). Loudness Judgement for Noise, Quality Judgement for Clarity (for Speech), Quality Judgement for Noisiness and overall preference were assessed across the pairs for three types of noise. From the results, it can be seen that NR 'on' condition was preferred as against NR 'off' conditions for all the tasks across the three types of noise. Results from Ricketts and Hornsby [4] indicated that digital noise reduction (DNR) 'on' condition (noise reduction set to maximum) was selected significantly more often than the DNR 'off' condition (noise reduction set to off) regardless of noise level or microphone mode. Also, they found that this preference was seen with the low noise levels (positive SNRs of $+6 \mathrm{~dB}$ ).

In comparison across the gradation, the NR max was chosen whenever it was compared with NR min and NR med; and NR med was chosen as against NR min. Brons et al. [16] evaluated the perceptual effects of noise reduction at two different SNRs (-4 $\mathrm{dB}$ SNR and $+4 \mathrm{~dB}$ SNR) for speech babble. Paired comparisons were done to study the annoyance, speech naturalness and overall preference for four different hearing aids. The NR was set to maximum. The results indicated that the hearing aid noise reduction was able to reduce the annoyance of speech babble at $-4 \mathrm{~dB}$ SNR (all four hearing aids) and at $+4 \mathrm{~dB}$ SNR (three of the four hearing aids). At $-4 \mathrm{~dB}$ SNR, two of the four noise reduction systems reduced speech naturalness. However, in the present study, there was no significant difference across NR gradations in most of the judgment task for speech babble.

The results of the present study were incongruent with a study by Alcántara et al. [17], who measured SRTs with NR and did not find changes in quality or comfort with noise reduction 'on' at negative SNRs for speech-shaped noise. As Brons et al. [16] suggest, the noise reduction systems differ from each other in how strongly they reduce the noise annoyance and preserve speech naturalness. Hence, the noise reduction used in the present study may not be able to effectively reduce the noise and improve speech at $0 \mathrm{~dB}$ SNR for speech babble. Brons et al. [16] have remarked that at lower SNRs, the noise reduction algorithm finds difficult to differentiate between speech and noise and thus, the reduction of noise would be accompanied by distortion of speech. In addition, Boymans and Dreschler [5] have reported that NR 'on' was preferred over NR 'off' at $+5 \mathrm{~dB}$ SNR. Thus, at favorable SNRs, NR would aid in the perception of speech in noise.

\section{Conclusion}

The study aimed to evaluate the effect of different gradation levels of noise reduction (NR) algorithm in hearing aids with three types of noise, through acoustical and perceptual measures. WADA-SNR, PESQ, and EDI were used to analyze the acoustical aspects and quality judgment task using paired comparisons were used to evaluate the perceptual effects. From the results, it can be inferred that there is an effective reduction in the level of noise when NR of the hearing aid was enabled in noise only condition. This reduction is effective at maximum NR gradation than at medium or minimum gradations.

In speech in noise condition, the maximum NR gradation showed better output SNR (as measured through WADA-SNR) than medium and minimum gradations (i.e., maximum $>$ medium $>$ minimum). The maximum improvement (the difference between NR 'off' and NR max) in the output SNR was seen only for cafeteria noise $(5.15 \mathrm{~dB})$ and traffic noise $(5.83 \mathrm{~dB})$. However, the NR gradation did not have an impact on speech babble $(0.72$ $\mathrm{dB}$ ). This could be due to the fact that the other types of noise had non-speech like characteristics and speech babble had 
speech-like characteristics. Similarly, temporal variations of the speech in noise signal (measured through EDI) were better preserved at maximum NR gradation. It was also noted evidently that, the minimum NR gradation had the least effect on the noise, i.e., NR 'on' at minimum gradation was almost equivalent to NR 'off' position across all the types of noise.

Though the PESQ, which is a perceptual evaluation of the speech quality, showed no pattern in effects across the NR gradations, the subjective evaluations using the pairedcomparisons showed that the NR max was chosen whenever it was matched with NR min and NR med; and NR med was chosen as against NR min. However, there was no significant difference across NR gradations in most of the judgment tasks for speech babble. Thus, when NR is set to maximum, it would aid is improving the speech quality and reduce the noisiness of the overall signal and this is dependent on the type of noise in the environment. Since the effect of NR was similar and minimum for speech babble, there is a need to develop a separate NR algorithm for speech babble. However, as NR algorithms differ from the way they are implemented in each make of the HA, the results cannot be generalized.

\section{Acknowledgement}

We like to acknowledge the Director, All India Institute of Speech and Hearing (AIISH), Mysuru and HOD-Audiology, AIISH, Mysuru for providing the infrastructure and equipment for the stu

\section{References}

1. Bentler R, Chiou LK (2006) Digital Noise Reduction: An Overview. Trends Amplif 10(2): 67-82.

2. Kochkin S MarkeTrak VII (2007) Obstacles to adult non-user adoption of hearing aids. Hear J 60(4): 27-43.

3. Chung K (2004) Challenges and recent developments in hearing aids. Part I. Speech understanding in noise, microphone technologies, and noise reduction algorithms. Trends Amplif 8(3): 83-124.

4. Ricketts TA, Hornsby BW (2005) Sound quality measures for speech in noise through a commercial hearing aid implementing digital noise reduction. J Am AcadAudiol 16(5): 270-277.

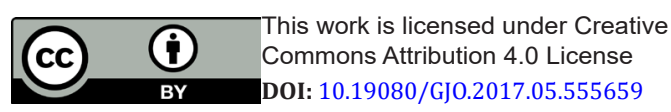

5. Boymans M, Dreschler WA (2000) Field trials using a digital hearing aid with active noise reduction and dual-microphone directionality. Audiol 39(5): 260-268.

6. Yuen KCP, Kam ACS, Lau PSH (2006) Comparative performance of an adaptive directional microphone system and a multichannel noise reduction system. J Am AcadAudiol 17(4): 241-252.

7. Zakis JA, Hau J, Blamey PJ (2009) Environmental noise reduction configuration - Effects on preferences, satisfaction, and speech understanding. Int J Audiol 48(12): 853-867.

8. Geetha C, Kumar SKS, Manjula P (2014) Development and standardization of the sentence identification test in the Kannada language. J Hg Sci 4(1): OA18-26.

9. Anitha R, Manjula P (2003) Effect of multi-speaker babble in different languages on speech recognition scores in Kannada. Mysuru: All India Institute of Speech and Hearing (Unpublished Independent project submitted to University of Mysuru).

10. Douglas Lanman (2006) Math code for Objective measures of speech quality/SNR. MATLAB Code.

11. Kim C, Stern RM, International Speech Communication Association (2008) Robust Signal-to-Noise Ratio Estimation Based on Waveform Amplitude Distribution Analysis, Brisbane, Australia, 22-26.

12. Ellis D (2011) Math code for Objective measures of speech quality/ SNR. MATLAB Code.

13. Fortune TW, Woodruff BD, Preves DA (1994) A new technique for quantifying temporal envelope contrasts. Ear Hear 15(1): 93-99.

14. Geetha C, Manjula P (2014) Effect of compression, digital noise reduction and directionality on envelope difference index, loglikelihood ratio and perceived quality. Audiol Res 4(1): 46-51.

15. Walaszek J (2012) Effects of compression in hearing aids on the envelope of the speech signal, Signal based measures of the sideeffects of the compression and their relation to speech intelligibility. Denmark: Technical University of Denmark; 2008. (Unpublished master's thesis). Cited in Souza P, Hoover E, Gallun F. Application of the Envelope Difference Index to spectrally sparse speech. J Speech Lang Hear Res 55(3): 824-837.

16. Brons I, Houben R, Dreschler WA (2013) Perceptual Effects of Noise Reduction with Respect to Personal Preference, Speech Intelligibility, and Listening Effort. Ear Hear 34(1): 29-41.

17. Alcántara JL, Moore BC, Kühnel V, Launer S (2003) Evaluation of the noise reduction system in a commercial digital hearing aid. Int J Audiol 42(1): 34-42.

\section{Your next submission with Juniper Publishers} will reach you the below assets

- Quality Editorial service

- Swift Peer Review

- Reprints availability

- E-prints Service

- Manuscript Podcast for convenient understanding

- Global attainment for your research

- Manuscript accessibility in different formats

( Pdf, E-pub, Full Text, Audio)

- Unceasing customer service

Track the below URL for one-step submission https://juniperpublishers.com/online-submission.php 